\title{
Systematic study for the mass distribution of fission fragments in the neutron rich region
}

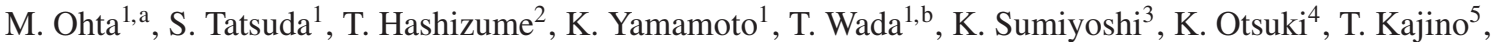 \\ H. Koura ${ }^{6}$, S. Chiba ${ }^{6}$, and T. Tachibana ${ }^{7}$ \\ 1 Department of Physics, Konan University Kobe 658-8501, Japan \\ 2 Allid Engineering Corporation, Tokyo, Japan \\ 3 Numazu College of Technology, Ooka 3600, Numazu 410-8501, Japan \\ 4 Department of Astronomy and Astrophysics, University of Chicago, USA \\ 5 National Astronomical Observatory, 2-21-1 Osawa, Mitaka, Tokyo 181-8588, Japan \\ 6 Advanced Science Research Center, JAEA Tokai, Naka, Ibaraki 319-1195, Japan \\ 7 Senior High School of Waseda University, 3-31-1 Kamishakujii, Nerima-ku, Tokyo 177-0044, Japan
}

\begin{abstract}
The systematic data on the fission fragment mass distribution for nuclei ranging from $Z=88$ to 120 relevant to the r-process nucleosynthesis is constructed in a semi-empirical way. The mass distribution is estimated from the potential energy surface by means of the liquid drop model with the shell energy correction and also by referring the results in the dynamical calculation of fission paths by the Langevin equation in the three-dimensional deformation space. The results are compared with the mass asymmetric values at the saddle point driven by the extended Thomas-Fermi Struchinsky integral (ETFSI) model. In the lighter nuclei $Z<100$, our results are consistent with the values of ETFSI, on the other hand in the heavier nuclei our estimation gives a larger mass asymmetry and approaches to zero due to the decrease of shell effect beyond $Z>120$.
\end{abstract}

\section{Introduction}

The importance of fission in the r-process nucleosynthesis has been frequently mentioned so far [1-4], but no consistent network calculation of the r-process including fission cycles is still realized yet. The role of fission in the r-process can be considered as follows: the fission process directly concerns with the abundances of transuranium isotopes, the fission products affect the formation of heavy nuclei in a wide mass range $70<A<180$ [5] depending on the r-process astrophysical sites, the fission process is a source of neutron density which controls the terminal point of the r-process and the mass distribution of heavy nuclei at the freeze-out stage, and the fission cycle is an unrevealed clue for more precise understanding of the r-process mechanism.

In order to challenge the consistent treatment in the r-process network calculation, several ingredients or the nuclear data inputs should be prepared. In the incorporation of fission process into the network, the fission barriers based on reliable mass table, the probability of neutron emission from the fission fragments, the neutron-induced fission rate, the beta-delayed fission rate, etc., and the fission fragment mass distribution are necessary.

In this paper, an attention is focused to the fission fragment mass distribution available for the r-process network calculation.

\footnotetext{
${ }^{a}$ Presenting author, e-mail: masaota@konan-u.ac.jp

${ }^{b}$ Present address: Department of Pure and Applied Physics, Kansai University, Suita, Osaka 564-8680, Japan
}

\section{Potential energy surface and the Langevin calculation}

As the method for estimating the fission fragment mass distribution, the use of the time dependent Schrödinger like equation [6] attracts attention for investigating the dynamical shape evolution of nucleus leading to the rupture. But the semimacroscopic approaches like as solving the stochastic equation (or the Langevin equation [7]) or studying the feature of the potential energy surface (PES) near the saddle-to-scission area [8] are rather suitable for obtaining the systematic data of the fission fragment mass distribution especially for the r-process network calculation. One example of the systematic analysis is a semi-empirical model of the fission fragment properties proposed by Benlliure et al. [9]. They parametrized the PES at the saddle point taking into account the shell effect of the fragments so as to reproduce the experimental mass distribution. Our method is similar in a sense using the PES, but is quite different. We investigate the PES near the scission point presenting in the shape parameter space suitable to enhance clearly the fission modes as describe later.

In the this work, the PES near the saddle and the scission point are calculated by means of the liquid drop model with the shell energy correction by the Two-Center shell model $[10,11]$ in the three-dimensional shape parameter space. The space is constituted from the distance between the center-ofmass of the fissioning fragments $z$, the deformation of the fragments $\delta$ (here the same deformation is assumed) and the mass asymmetry parameter $\alpha$.

The dynamical fusion and fission path in the deformation space is given so far by solving the Langevin equation [12-16] which is a set of six differential equations for the generalized 


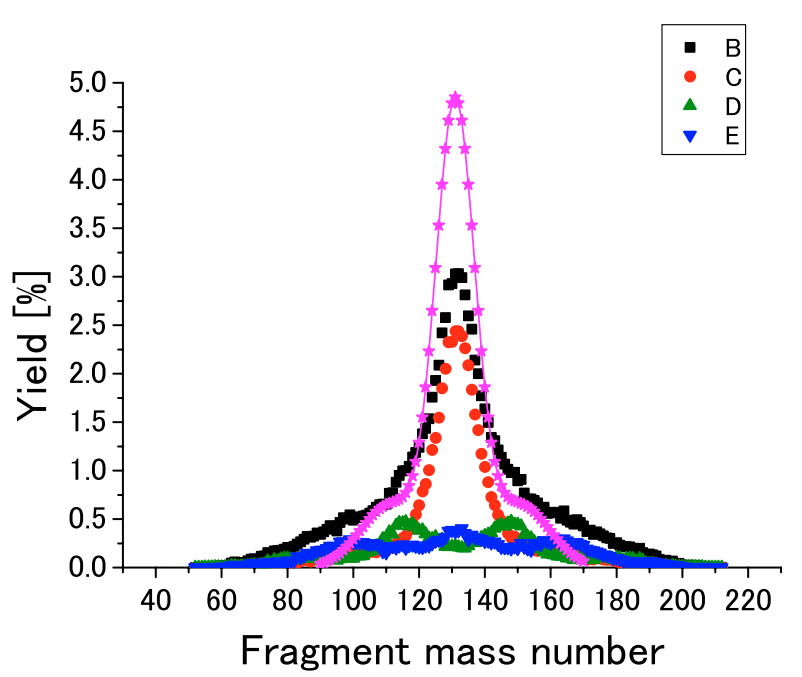

Fig. 1. Fission fragment mass distribution obtained by the Langevin calculation [21] for ${ }^{264} \mathrm{Fm}$. Solid squares (black, B) show the total mass distribution and the partial distributions corresponding to three fission modes (C, D, E) are also drawn. Solid stars (violet) show the present parametrization.

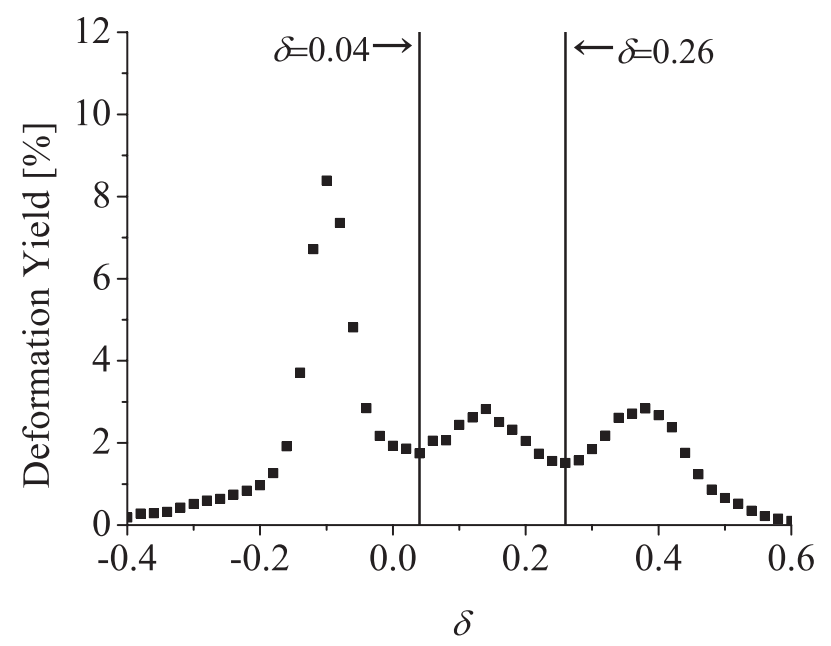

Fig. 2. Distribution of the fragment deformation $\delta$ [21]. Three prominent peakes are corresponding to three different fission mode.

coordinate and momentum corresponding to $z, \alpha$ and $\delta$. In the Langevin equation, the hydro-dynamical mass tensor by Werner-Wheeler [17], the wall-and-window one-body dissipation tensor $[18,19]$ and the random force whose strength satisfies the classical fluctuation-dissipation theorem are used. For several actinide nuclei, Ichikawa [20] and Asano [21] have calculated the fission paths for the purpose of the analysis of fission modes.

Typical example for ${ }^{264} \mathrm{Fm}$ is shown in figures 1 and 2 . In figure 1, the fission fragment mass distribution and its partial distributions corresponding to three fission modes are shown. The distribution of the $\delta$ parameter at the scission point is depicted in figure 2. The striking feature in figure 2 is a clear separation of fission mode, i.e., the symmetric component around $\delta \sim 0$ (standard I) and the asymmetric ones around $\delta \sim 0.15$ and $\sim 0.25$ (standard II, etc.). The presentation of yields in the $\delta$ - coordinate is very useful to see the fission
Table 1. Weight factor for the symmetric component $w_{s}$.

\begin{tabular}{llllll}
\hline $\mathrm{N}$ & $\mathrm{Z}=92$ & $\mathrm{Z}=93$ & $\mathrm{Z}=94$ & $\mathrm{Z}=95$ & $\mathrm{Z}=96$ \\
\hline 158 & 0 & 0 & 0 & 0 & 0 \\
159 & 0 & 0 & 0 & 0 & 0.001 \\
160 & 0 & 0 & 0 & 0.048 & 0.007 \\
161 & 0 & 0.009 & 0.049 & 0.112 & 0.132 \\
162 & 0 & 0.044 & 0.089 & 0.156 & 0.192 \\
163 & 0.097 & 0.174 & 0.219 & 0.286 & 0.331 \\
164 & 0.152 & 0.208 & 0.252 & 0.331 & 0.369 \\
165 & 0.043 & 0.089 & 0.14 & 0.209 & 0.244 \\
166 & 0 & 0.017 & 0.06 & 0.123 & 0.148 \\
167 & 0 & 0 & 0 & 0.001 & 0.025 \\
168 & 0 & 0 & 0 & 0 & 0 \\
169 & 0 & 0 & 0 & 0 & 0.068 \\
170 & 0 & 0 & 0 & 0 & 0.033 \\
171 & 0 & 0 & 0 & 0 & 0 \\
\hline
\end{tabular}

mode. We can see the definite relation between this structure and the position of the valley in the PES near scission, when we draw the PES in the $\alpha-\delta$ plane.

By referring the results given by Ichikawa [20] and Asano [21], we planned to have a systematic analysis of the PES to make the data set on the fission fragment mass distribution in which both the symmetric and the asymmetric component are taken into account without details on the identification to various asymmetric modes. The guidelines of our estimation for the mass asymmetric parameter are the use of the PES in the $\alpha-\delta$ plane, and the quantification of the depth of valley which has strong correlation with the thickness of the fission path or the measure of the mixture of different modes. The rule to determine the asymmetric parameter from the PES is then as follows: for the asymmetric component the average value of $\alpha$ corresponding to the position of valley at $\delta=0.2$ and 0.3 is adopted where the substantial part of fission fragments fall in, and for the symmetric component the contribution is determined by comparing the depth of the PES at $\delta \sim 0$ and $\alpha=0$ with the depth of the asymmetric valley. The fission fragment mass distribution by our parameter set (solid violet stars in fig. 1) is compared with the Langevin calculation (solid black squares marked B) in figure 1. Details in the asymmetric modes (D, E in fig. 1) are treated in an averaged manner.

\section{Data set of the fission mass asymmetry}

The mass asymmetries for the isotopes of five nuclei with $\mathrm{Z}=92 \sim 96$ are listed in table 2 . The value with the asterisk means that the mass distribution has the symmetric component with the weight factor $w_{s}$ listed in table 1 . The mixture of the symmetric component comes from the strong shell effect of the fission product having double magic property ${ }_{50}^{132} \mathrm{Sn}$. Therefore, the weight of the symmetric component is maximum for ${ }^{264} \mathrm{Fm}$ whose value is 0.71 . (One can get the full data set from the web site http://tpweb2.phys.konanu.ac.jp/en/fission.html.)

The mass asymmetry of the fission products is not simply determined from the mass asymmetry of the saddle point. One 
Table 2. Fission fragment mass asymmetry for isotopes of nuclei with $\mathrm{Z}=92 \sim 96$. Isotopes with the asterisk value have an additional symmetric component with the weight factor listed in table 1 .

\begin{tabular}{|c|c|c|c|c|c|}
\hline $\mathrm{N}$ & $Z=92$ & $Z=93$ & $Z=94$ & $Z=95$ & $Z=96$ \\
\hline 138 & 0.1 & 0.075 & 0.1 & 0.1 & 0.1 \\
\hline 139 & 0.075 & 0.075 & 0.1 & 0.075 & 0.1 \\
\hline 140 & 0.125 & 0.1 & 0.125 & 0.1 & 0.1 \\
\hline 141 & 0.1 & 0.1 & 0.125 & 0.075 & 0.15 \\
\hline 142 & 0.125 & 0.125 & 0.15 & 0.1 & 0.15 \\
\hline 143 & 0.15 & 0.125 & 0.15 & 0.1 & 0.15 \\
\hline 144 & 0.15 & 0.15 & 0.15 & 0.1 & 0.15 \\
\hline 145 & 0.15 & 0.15 & 0.15 & 0.15 & 0.15 \\
\hline 146 & 0.15 & 0.15 & 0.15 & 0.15 & 0.15 \\
\hline 147 & 0.15 & 0.15 & 0.15 & 0.15 & 0.15 \\
\hline 148 & 0.15 & 0.15 & 0.15 & 0.15 & 0.15 \\
\hline 149 & 0.15 & 0.15 & 0.15 & 0.15 & 0.15 \\
\hline 150 & 0.15 & 0.15 & 0.15 & 0.15 & 0.15 \\
\hline 151 & 0.15 & 0.15 & 0.15 & 0.15 & 0.15 \\
\hline 152 & 0.15 & 0.15 & 0.15 & 0.15 & 0.15 \\
\hline 153 & 0.15 & 0.175 & 0.15 & 0.15 & 0.175 \\
\hline 154 & 0.15 & 0.175 & 0.15 & 0.15 & 0.175 \\
\hline 155 & 0.125 & 0.15 & 0.15 & 0.15 & 0.15 \\
\hline 156 & 0.125 & 0.15 & 0.175 & 0.15 & 0.175 \\
\hline 157 & 0.125 & 0.15 & 0.15 & 0.15 & 0.15 \\
\hline 158 & 0.15 & 0.15 & 0.15 & 0.15 & 0.15 \\
\hline 159 & 0.15 & 0.15 & 0.15 & 0.15 & $0.15^{*}$ \\
\hline 160 & 0.1 & 0.15 & 0.15 & $0.15^{*}$ & $0.15 *$ \\
\hline 161 & 0.1 & $0.15^{*}$ & $0.15^{*}$ & $0.15^{*}$ & $0.15^{*}$ \\
\hline 162 & 0.1 & $0.15^{*}$ & $0.15^{*}$ & $0.15^{*}$ & $0.15^{*}$ \\
\hline 163 & $0.1^{*}$ & $0.2 *$ & $0.15^{*}$ & $0.15^{*}$ & $0.15^{*}$ \\
\hline 164 & $0.1^{*}$ & $0.2^{*}$ & $0.15^{*}$ & $0.15^{*}$ & $0.15^{*}$ \\
\hline 165 & $0.1^{*}$ & $0.2 *$ & $0.1^{*}$ & $0.125^{*}$ & $0.1^{*}$ \\
\hline 166 & 0.1 & $0.2^{*}$ & $0.1^{*}$ & $0.125^{*}$ & $0.1^{*}$ \\
\hline 167 & 0.1 & 0.225 & 0.125 & $0.125^{*}$ & $0.175^{*}$ \\
\hline 168 & 0.075 & 0.2 & 0.1 & 0.2 & 0.175 \\
\hline 169 & 0.075 & 0.15 & 0.15 & 0.175 & $0.15^{*}$ \\
\hline 170 & 0.075 & 0.125 & 0.15 & 0.2 & $0.175^{*}$ \\
\hline 171 & 0.075 & 0.175 & 0.15 & 0.175 & 0.15 \\
\hline 172 & 0.075 & 0.15 & 0.15 & 0.175 & 0.15 \\
\hline 173 & 0.075 & 0.15 & 0.15 & 0.175 & 0.15 \\
\hline 174 & 0.075 & 0.15 & 0.15 & 0.175 & 0.15 \\
\hline 175 & 0.075 & 0.15 & 0.15 & 0.175 & 0.175 \\
\hline 176 & 0.075 & 0.15 & 0.15 & 0.175 & 0.15 \\
\hline 177 & 0.075 & 0.15 & 0.15 & 0.175 & 0.175 \\
\hline 178 & 0.075 & 0.15 & 0.15 & 0.175 & 0.175 \\
\hline 179 & 0.075 & 0.075 & 0.15 & 0.175 & 0.175 \\
\hline 180 & 0.075 & 0.125 & 0.15 & 0.2 & 0.175 \\
\hline 181 & 0.05 & 0.1 & 0.125 & 0.15 & 0.175 \\
\hline 182 & 0.075 & 0.125 & 0.125 & 0.175 & 0.175 \\
\hline 183 & 0.075 & 0.125 & 0.125 & 0.175 & 0.125 \\
\hline 184 & 0.075 & 0.125 & 0.125 & 0.175 & 0.125 \\
\hline 185 & 0.075 & 0.125 & 0.125 & 0.175 & 0.125 \\
\hline 186 & 0.075 & 0.05 & 0.125 & 0.175 & 0.1 \\
\hline 187 & 0.05 & 0.05 & 0.075 & 0.1 & 0.1 \\
\hline 188 & 0.05 & 0.075 & 0.1 & 0.1 & 0.1 \\
\hline 189 & & 0.05 & 0.1 & 0.075 & 0.1 \\
\hline 190 & & 0.075 & 0.1 & 0.075 & 0.1 \\
\hline 191 & & 0.075 & 0.075 & 0.075 & 0.1 \\
\hline 192 & & 0.075 & 0.1 & 0.075 & 0.1 \\
\hline 193 & & 0.075 & 0.075 & 0.075 & 0.075 \\
\hline
\end{tabular}

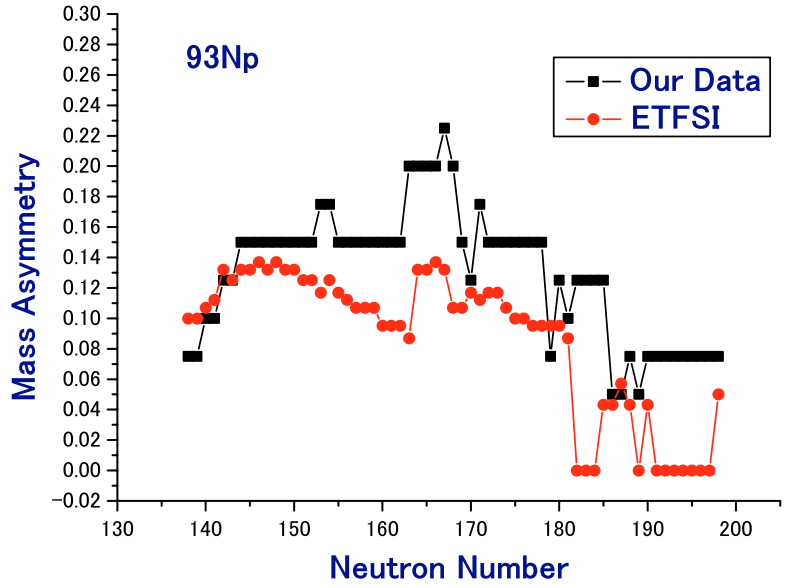

Fig. 3. Comparison of our asymmetry parameters with that derived from ETFSI data [22] for $\mathrm{Np}$ isotopes.

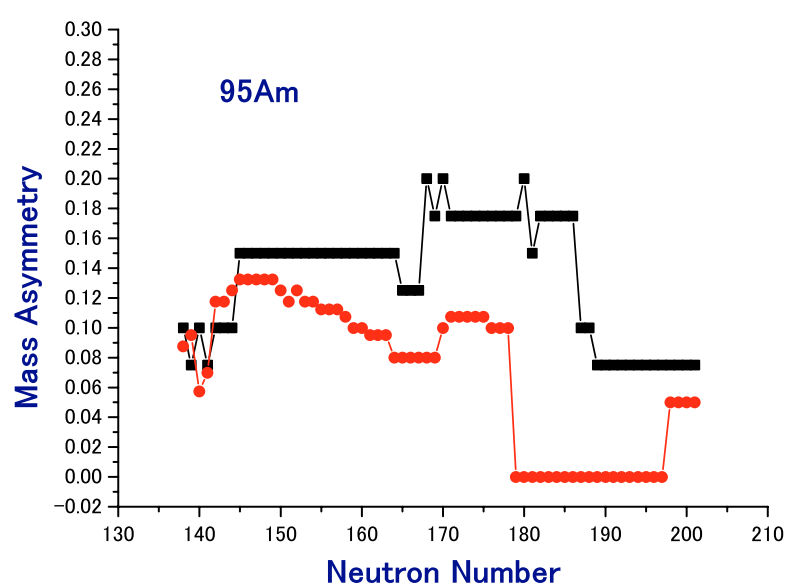

Fig. 4. Comparison of our asymmetry parameters with that derived from ETFSI data [22] for Am isotopes.

should investigate the dynamic shape evolution of fissioning system from the saddle to the scission point. The mass asymmetry is modified according to the feature of the valley extending to the scission point and the strength of the nuclear friction is also the important factor. However, the comparison of our asymmetry with that given by the value of the saddle point calculated by the ETFSI model [22] is meaningful, since the property of the asymmetry at the saddle point is still kept beyond the saddle. Figure 3 shows the comparison of our mass asymmetry of $\mathrm{Np}$ isotopes (black solid squares) with that extracted from the saddle information of ETFSI model [22] (red solid circles). The trend of both data is very similar. This means that the PES calculated by the two different models have similar structure of valley from the saddle to the scission point. The same comparison for Am isotopes is shown in figure 4. In this case, the deviation in the large neutron number can be seen. Such deviation becomes larger when the atomic number increases. It is found that the PES of our model in the neutron rich region still has prominent valley toward the scission point due to the shell effect. However, for $Z>120$ the asymmetry parameters become less than $\sim 0.05$ due to the disappearance of the shell effect in the fragments. 

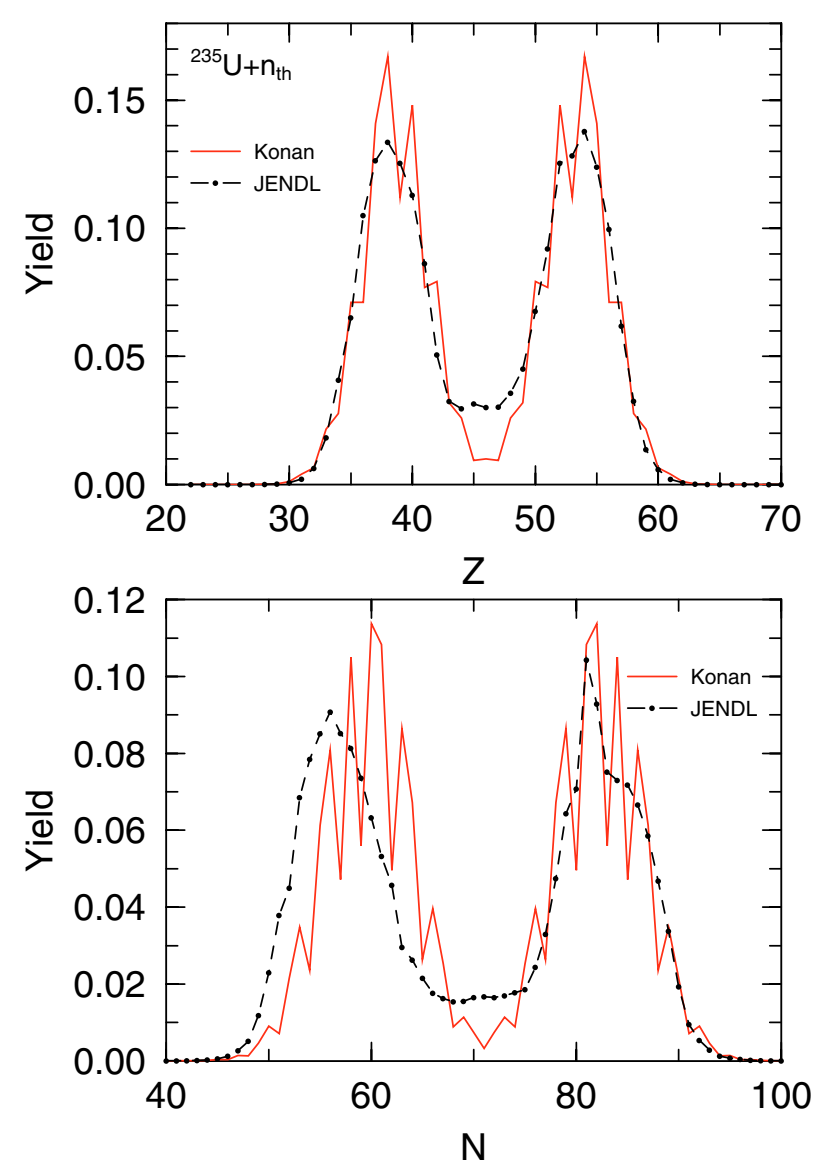

Fig. 5. Fission fragment mass distribution for ${ }^{236} \mathrm{U}$. The present work (red line) is compared with the data from JENDL [23].

Once we obtains the peak position of the fragment mass distribution with above mentioned way, the whole fragment mass distribution $f(A)$ is derived by assuming the Gaussian distribution around the most probable heavy $(\mathrm{H})$ and light $(\mathrm{L})$ fragment mass $A_{H}$ and $A_{L}$ for the asymmetric fission and $A / 2$ for the symmetric case.

$$
\begin{aligned}
f(A)= & \frac{1}{\sqrt{2 \pi} \sigma}\left(1-w_{s}\right)\left(e^{-\left(A_{H}-A\right)^{2} / 2 \sigma^{2}}+e^{-\left(A_{L}-A\right)^{2} / 2 \sigma^{2}}\right) \\
& +2 w_{s} \frac{1}{\sqrt{2 \pi} \sigma} e^{-\left(\left(A-N_{\text {loss }}\right) / 2-A\right)^{2} / 2 \sigma^{2}} .
\end{aligned}
$$

The value of the dispersion parameter $\sigma$ is determined from the result of the Langevin calculation and is set tentatively to 7.0 in the present work. For a given mass asymmetry $\alpha$, $A_{H}=(1+\alpha)\left(A-N_{\text {loss }}\right) / 2$ and $A_{L}=(1-\alpha)\left(A-N_{\text {loss }}\right) / 2$, here $N_{\text {loss }}=2$ is assumed. The division of protons is done so as to keep the proton to neutron ratio of the fissioning nuclei: $\left.Z / N=Z_{H} /\left(N_{H}+\left(N_{\text {loss }} / 2\right)\right)=Z_{L} /\left(N_{L}+N_{\text {loss }} / 2\right)\right)$. The example in ${ }^{236} \mathrm{U}$ is presented in figure 5 . The notches appeared in the red line is the computational origin in the separation of $A$ into $Z$ and $N$.

\section{Summary and perspectives}

Referring the results of the Langevin calculation on the fission path, we have estimated the fission mass asymmetry and have constructed the data set. It is noted that the investigation of the PES presented in the $\alpha-\delta$ space helps the systematic analysis to determine the mass asymmetry, because the fragment deformation $\delta$ correlates strongly to the fission mode.

The present data set is incorporated into the r-process network code and we begin to discuss the preliminary results on the effect of fission cycle and so on. We expect that the calculation taking account of the fragment mass distribution will add to an interesting new understanding on the r-process element abundance so far discussed.

\section{References}

1. J.J. Cowan, F.-K. Thielemann, J.W. Truran, Phys. Rep. 208, 267 (1991).

2. I.V. Panov, E. Kolbe, B. Pfeiffer, T. Rauscher, K.-L. Kratz, F.-K. Thielmenn, Nucl. Phys. A 747, 633 (2005).

3. B. Pfeiffer, K.-L. Kratz, F.-K. Thielemann, W.B. Walters, Nucl. Phys. A 693, 282 (2001).

4. T. Rauscher, J.H. Applegate, J.J. Cowan, F.-K. Thielemann, M. Wiescher, ApJ. 429, 499 (1994).

5. S. Chiba et al., in Workshop on the fission cycle in the r-process, 23 March 2007, NAO Mitaka Tokyo (private communication).

6. H. Goutte, J.-F. Berger, P. Casoli, D. Gogny, Phys. Rev. C 71, 024316 (2005).

7. T. Wada, Y. Abe, N. Carjan, Phys. Rev. Lett. 70, 3538 (1993).

8. V.V. Pashkevich, Nucl. Phys. A 169, 275 (1971).

9. J. Benlliure, A. Greve, M. de Jong, K.-H. Schmidt, S. Zhhanov, Nucl. Phys. A 628, 456 (1998).

10. A. Iwamoto, S. Yamaji, S. Suekane, K. Harada, Prog. Theor. Phys. 55, 115 (1976).

11. K. Sato, S. Yamaji, K. Harada, S. Yoshida, Z. Phys. A 290, 149 (1979).

12. T. Tokuda, T. Wada, M. Ohta, Prog. Theor. Phys. 101, 607 (1999).

13. T. Wada, Y. Aritomo, T. Tokuda, K. Okazaki, M. Ohya, Y. Abe, Nucl. Phys. A 654, 888c (1999).

14. Y. Aritomo M. Ohta, Nucl. Phys. A 744, 3 (2004).

15. Y. Aritomo, M. Ohta, T. Materna, F. Hanappe, O. Dorvaux, L. Stuttge, Nucl. Phys. A 759, 309 (2005).

16. Y. Aritomo, M. Ohta, Nucl. Phys. A 764, 149 (2006).

17. K.T.R. Davies, A.J. Sierk, J.R. Nix, Phys. Rev. C 13, 2385 (1976).

18. J. Blocki, Y. Bonen, J.R. Nix, J. Randrup, M. Robel, A.J. Sierk, W.S. Swiatecki, Ann. Phys. 113, 330 (1978).

19. H. Feldmeier, Rep. Prog. Phys. 50, 915 (1987).

20. T. Ichikawa, T. Asano, T. Wada, M. Ohta, J. Nucl. Radiochem. Sci. 3, 67 (2002).

21. T. Asano, T. Wada, M. Ohta, T. Ichikawa, S. Yamaji, H. Nakahara, J. Nucl. Radiochem. Sci. 5, 1 (2004).

22. A. Mamdouh, J.M. Pearson, M. Rayet, F. Tondeur, Nucl. Phys. A 679, 337 (2001).

23. K. Tasaka et al., JNDC Nuclear Data Library of Fission Products, Second Version, JAERI (1990) 1320. 